\title{
EventCap: Monocular 3D Capture of High-Speed Human Motions using an Event Camera
}

\author{
Lan $\mathrm{Xu}^{1,2,3}$ Weipeng $\mathrm{Xu}^{2}$ Vladislav Golyanik ${ }^{2}$ Marc Habermann $^{2}$ Lu Fang $^{1}$ Christian Theobalt ${ }^{2}$ \\ ${ }^{1}$ Tsinghua-Berkeley Shenzhen Institute, Tsinghua University, China \\ ${ }^{2}$ Max Planck Institute for Informatics, Saarland Informatics Campus, Germany \\ ${ }^{3}$ Robotics Institute, Hong Kong University of Science and Technology, Hong Kong
}

\begin{abstract}
The high frame rate is a critical requirement for capturing fast human motions. In this setting, existing markerless image-based methods are constrained by the lighting requirement, the high data bandwidth and the consequent high computation overhead. In this paper, we propose EventCap - the first approach for $3 D$ capturing of high-speed human motions using a single event camera. Our method combines model-based optimization and CNNbased human pose detection to capture high-frequency motion details and to reduce the drifting in the tracking. As a result, we can capture fast motions at millisecond resolution with significantly higher data efficiency than using high frame rate videos. Experiments on our new event-based fast human motion dataset demonstrate the effectiveness and accuracy of our method, as well as its robustness to challenging lighting conditions.
\end{abstract}

\section{Introduction}

With the recent popularity of virtual and augmented reality (VR and AR), there has been a growing demand for reliable 3D human motion capture. As a low-cost alternative to the widely used marker and sensor-based solutions, markerless video-based motion capture alleviates the need for intrusive body-worn motion sensors and markers. This research direction has received increased attention over the last years [13, 21, 54, 64, 68].

In this paper, we focus on markerless motion capture for high-speed movements, which is essential for many applications such as training and performance evaluation for gymnastics, sports and dancing. Capturing motion at a high frame rate leads to a very high data bandwidth and algorithm complexity for the existing methods. While the current marker and sensor-based solutions can support more than 400 frames per second (fps) [63, 66, 44], the literature on markerless high frame rate motion capture is sparse.

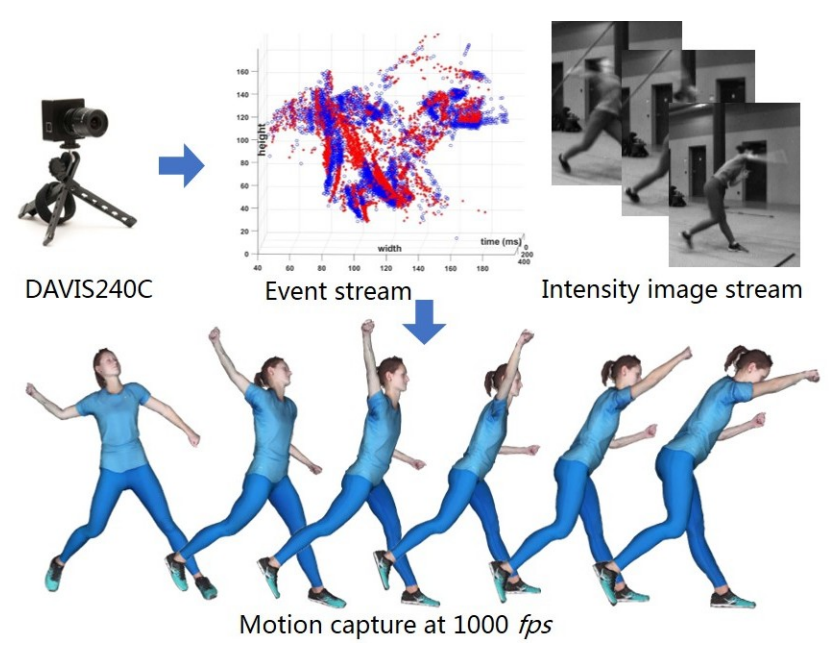

Figure 1: We present the first monocular event-based 3D human motion capture approach. Given the event stream and the low frame rate intensity image stream from a single event camera, our goal is to track the highspeed human motion at 1000 frames per second.

Several recent works $[30,71]$ revealed the importance of the high frame rate camera systems for tracking fast motions. However, they still suffer from the aforementioned fundamental problem - the high frame rate leads to excessive amounts of raw data and large bandwidth requirement for data processing (e.g., capturing RGB stream of VGA resolution at $1000 \mathrm{fps}$ from a single view for one minute yields 51.5GB of data). Moreover, both methods [30, 71] assume 1) well-lit scenarios for compensating the short exposure time at high frame rate, and 2) indoor capture due to the limitation of the IR-based depth sensor.

In this paper, we propose a rescue to the problems outlined above by using an event camera. Such bio-inspired dynamic vision sensors [32] asynchronously measure perpixel intensity changes and have multiple advantages over conventional cameras, including high temporal resolution, high dynamic range (140dB), low power consumption and low data bandwidth. These properties potentially allow capturing very fast motions with high data efficiency and in 
general lighting conditions. Nevertheless, using the event camera for motion capture is still challenging. First, the high temporal resolution of the algorithm leads to very sparse measurements (events) in each frame interval, since the inter-frame intensity changes are subtle. The resulting low signal-to-noise ratio (SNR) makes it difficult to track the motion robustly. Second, since the event stream only encodes temporal intensity changes, it is difficult to initialize the tracking and prevent drifting. A naïve solution is to reconstruct images at a high frame rate by accumulating the events and apply existing methods on the reconstructed images. Such a policy makes the data dense again, and the temporal information encoded in the events is lost.

To tackle these challenges, we propose EventCap - the first monocular event-based 3D human motion capture approach (see Fig. 1 for an overview). More specifically, we design a hybrid and asynchronous motion capture algorithm that leverages the event stream and the low frame rate intensity image stream from the event camera in a joint optimization framework. Our method consists of three stages: First, we track the events in 2D space in an asynchronous manner and reconstruct the continuous spatio-temporal event trajectories between each adjacent intensity images. By evenly slicing the continuous event trajectories, we achieve 2D event tracking at the desired high frame rate. Second, we estimate the 3D motion of the human actor using a batchbased optimization algorithm. To tackle drifting due to the accumulation of tracking errors and depth ambiguities inherent to the monocular setting, our batch-based optimization leverages not only the tracked event trajectories but also the CNN-based 2D and 3D pose estimation from the intensity images. Finally, we refine the captured high-speed motion based on the boundary information obtained from the asynchronous event stream. To summarise, the main contributions of this paper include:

- We propose the first monocular approach for event camera-based 3D human motion capture.

- To tackle the challenges of low signal-to-noise ratio (SNR), drifting and the difficulty in initialization, we propose a novel hybrid asynchronous batch-based optimization algorithm.

- We propose an evaluation dataset for event camerabased fast human motion capture and provide highquality motion capture results at $1000 \mathrm{fps}$. The dataset will be publicly available.

\section{Related Work}

3D Human Motion Capture. Marker-based multi-view motion capture studios are widely used in both industry and academia [66, 63, 44], which can capture fast motions at high frame rate (e.g., 960 fps) [44]. Those systems are usually costly, and it is quite intrusive for the users to wear the marker suites. Markerless multicamera motion capture algorithms overcome these problems [5, 58, 37, 22, 16, 51, 52, 54, 25, 67]. Recent work [2, 6, 14, 47, 48, 42, 53] even demonstrates robust out-ofstudio motion capture. Although the cost is drastically reduced, synchronizing and calibrating multi-camera systems is still cumbersome. Furthermore, when capturing fast motion at high frame rate [30], a large amount of data from multiple cameras becomes a bottleneck not only for the computation but also for data processing and storage.

The availability of commodity depth cameras enabled low-cost motion capture without complicated multi-view setups [50, 3, 65, 70, 19]. To capture fast motions, Yuan et al. [71] combine a high frame rate action camera with a commodity $30 \mathrm{fps}$ RGB-D camera, resulting in a synthetic depth camera of $240 \mathrm{fps}$. However, the active IR-based cameras are unsuitable for outdoor capture, and their high power consumption limits the mobile application.

Recently, purely RGB-based monocular 3D human pose estimation methods have been proposed with the advent of deep neural networks [23, 49, 11, 61, 29]. These methods either regress the root-relative $3 \mathrm{D}$ positions of body joints from single images [31, 56, 72, 34, 57, 41, 35], or lift 2D detection to 3D [4, 73, 10, 69, 24]. The 3D positional representation used in those works is not suitable for animating 3D virtual characters. To solve this problem, recent works regress joint angles directly from the images [26, 28, 39, 43, 55]. In theory, these methods can be applied directly on high frame rate video for fast motion capture. In practice, the tracking error is typically larger than the inter-frame movements, which leads to the loss of finelevel motion details. Methods combining data-driven 3D pose estimation and image-guided registration alleviate this problem and can achieve higher accuracy [68, 20]. However, data redundancy is still an issue.

Furthermore, when capturing a high frame rate RGB video, the scene has to be well-lit, since the exposure time cannot be longer than the frame interval. Following [68], we combine data-driven method with batch optimization. Differently, instead of using high frame rate RGB video, we leverage the event stream and the low frame rate intensity image stream from an event camera. Compared to RGB-based methods, our approach is more data-efficient and works well in a broader range of lighting conditions.

Tracking with Event Cameras. Event cameras are causing a paradigm shift in computer vision, due to their high dynamic range, absence of motion blur and low power consumption. For a detailed survey of the event-based vision applications, we refer to [17]. The most closely related settings to ours are found in works on object tracking from an event stream. 


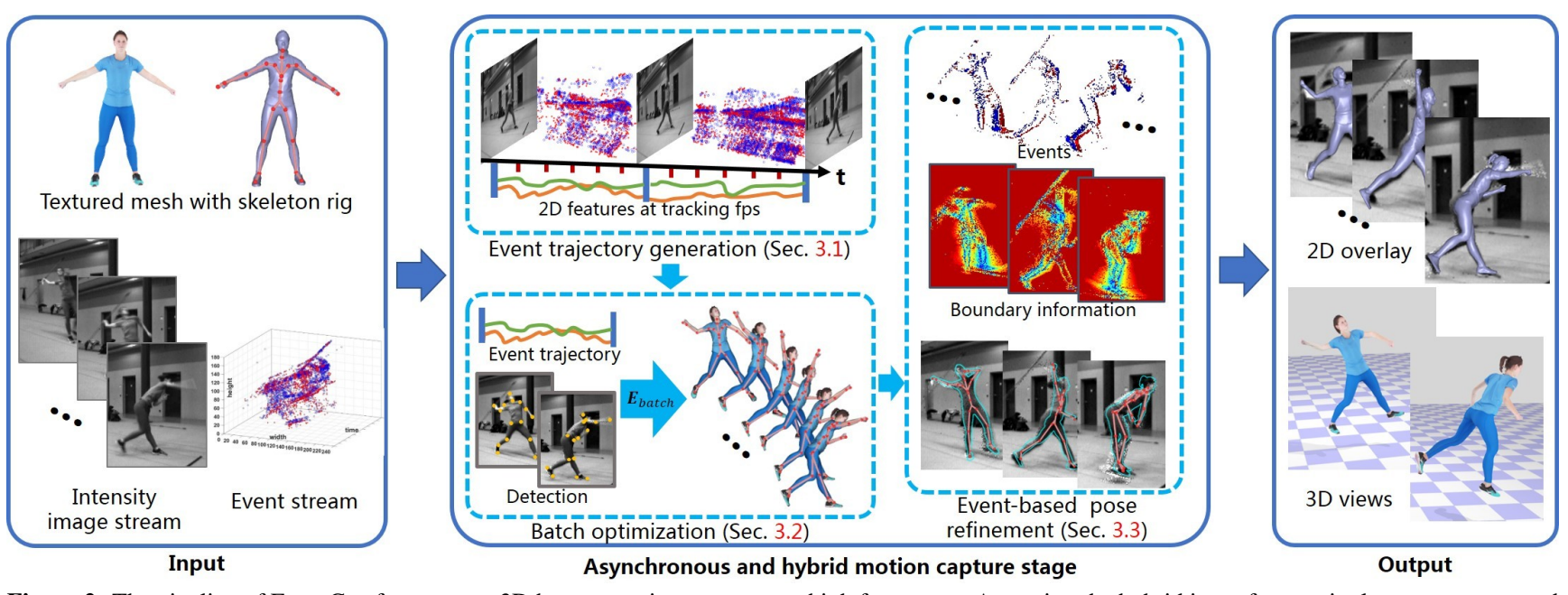

Figure 2: The pipeline of EventCap for accurate 3D human motion capture at a high frame rate. Assuming the hybrid input from a single event camera and a personalized actor rig, we first generate asynchronous event trajectories (Sec. 3.1). Then, the temporally coherent per-batch motion is recovered based on both the event trajectories and human pose detections (Sec. 3.2). Finally, we perform event-based pose refinement (Sec. 3.3).

The specific characteristics of the event camera make it very suitable for tracking fast moving objects. Most of the related works focus on tracking $2 \mathrm{D}$ objects like known 2D templates [38, 36], corners [62] and lines [15]. Piatkowska et al. [45] propose a technique for multi-person bounding box tracking from a stereo event camera. Valeiras et al. [60] track complex objects like human faces with a set of Gaussian trackers connected with simulated springs.

The first 3D tracking method was proposed in [46], which estimates the 3D pose estimation of rigid objects. Starting from a known object shape in a known pose, their method incrementally updates the pose by relating events to the closest visible object edges. Recently, Calabrese et $a l$. [7] provide the first event-based 3D human motion capture method based on multiple event cameras. A neural network is trained to detect 2D human body joints using the event stream from each view. Then, the $3 \mathrm{D}$ body pose is estimated through triangulation. In their method, the events are accumulated over time, forming image frames as input to the network. Therefore, the asynchronous and high temporal resolution natures of the event camera are undermined, which prevents the method from being used for high frame rate motion capture.

\section{EventCap Method}

Our goal in this paper is to capture high-speed human motion in 3D using a single event camera. In order to faithfully capture the fine-level details in the fast motion, a high temporal resolution is necessary. Here, we aim at a tracking frame rate of $1000 \mathrm{fps}$.

Fig. 2 provides an overview of EventCap. Our method relies on a pre-processing step to reconstruct a template mesh of the actor. During tracking, we optimize the skele- ton parameters of the template to match the observation of a single event camera, including the event stream and the low frame rate intensity image stream. Our tracking algorithm consists of three stages: First, we generate sparse event trajectories between two adjacent intensity images, which extract the asynchronous spatio-temporal information from the event stream (Sec. 3.1). Then, a batch optimization scheme is performed to optimize the skeletal motion at $1000 \mathrm{fps}$ using the event trajectories and the CNNbased body joint detection from the intensity image stream (Sec. 3.2). Finally, we refine the captured skeletal motion based on the boundary information obtained from the asynchronous event stream (Sec. 3.3).

Template Mesh Acquisition. We use a 3D body scanners [59] to generate the template mesh of the actor. To rig the template mesh with a parametric skeleton, we fit the Skinned Multi-Person Linear Model (SMPL)[33] to the template mesh by optimizing the body shape and pose parameters, and then transfer the SMPL skinning weights to our scanned mesh. One can also use image-based human shape estimation algorithms, e.g. [26], to obtain a SMPL mesh as the template mesh, if the 3D scanner is not available. A comparison of these two methods is provided in Sec. 4.1. To resemble the anatomic constraints of body joints, we reduce the degrees of freedom of the SMPL skeleton. Our skeleton parameter set $\mathbf{S}=[\boldsymbol{\theta}, \mathbf{R}, \mathbf{t}]$ includes the joint angles $\boldsymbol{\theta} \in \mathbb{R}^{27}$ of the $N_{J}$ joints of the skeleton, the global rotation $\mathbf{R} \in \mathbb{R}^{3}$ and translation $\mathbf{t} \in \mathbb{R}^{3}$ of the root.

Event Camera Model. Event cameras are bio-inspired sensors that measure the changes of logarithmic brightness $\mathcal{L}(u, t)$ independently at each pixel and provide an asynchronous event stream at microsecond resolution. An event $e_{i}=\left(u_{i}, t_{i}, \rho_{i}\right)$ is triggered at pixel $u_{i}$ at time $t_{i}$ when the logarithmic brightness change reaches a thresh- 
old: $\mathcal{L}\left(u_{i}, t_{i}\right)-\mathcal{L}\left(u_{i}, t_{p}\right)=p_{i} C$, where $t_{p}$ is the timestamp of the last event occurred at $u_{i}, p_{i} \in\{-1,1\}$ is the event polarity corresponding to the threshold $\pm C$. Besides the event stream, the camera also produces an intensity image stream at a lower frame rate, which can be expressed as an average of the latent images during the exposure time:

$$
\mathcal{I}(k)=\frac{1}{T} \int_{t_{k}-T / 2}^{t_{k}+T / 2} \exp (\mathcal{L}(t)) d t,
$$

where $t_{k}$ is the central timestamp of the $k$-th intensity image and $T$ is the exposure time. Note that $\mathcal{I}(k)$ can suffer from severe motion blur due to high-speed motions.

\subsection{Asynchronous Event Trajectory Generation}

A single event does not carry any structural information and therefore tracking based on isolated events is not robust. To extract the spatio-temporal information from the event stream, in the time interval $\left[t_{k}, t_{k+1}\right]$ (denoted as the $k$-th batch) between adjacent intensity images $\mathcal{I}(k)$ and $\mathcal{I}(k+1)$, we use [18] to track the photometric $2 \mathrm{D}$ features in an asynchronous manner, resulting in the sparse event trajectories $\{\mathcal{T}(h)\}$. Here, $h \in[1, H]$ denotes the temporal 2D pixel locations of all the $H$ photometric features in the current batch, which are further utilized to obtain correspondences to recover high-frequency motion details.

Intensity Image Sharpening. Note that [18] relies on sharp intensity images for gradient calculation. However, the intensity images suffer from severe motion blur due to the fast motion. Thus, we first adopt the event-based double integral (EDI) model [40] to sharpen the images $\mathcal{I}(k)$ and $\mathcal{I}(k+1)$. A logarithmic latent image $\mathcal{L}(t)$ can be formulated as $\mathcal{L}(t)=\mathcal{L}\left(t_{k}\right)+\mathcal{E}(t)$, where $\mathcal{E}(t)=\int_{t_{k}}^{t} p_{i}(s) C \delta(s) d s$ denotes continuous event accumulation. By aggregating the latent image $\mathcal{I}(k)$ (see Eq. (1)) and the logarithmic intensity changes, we obtain the sharpened image:

$\mathcal{L}\left(t_{k}\right)=\log (\mathcal{I}(k))-\log \left(\frac{1}{T} \int_{t_{k}-T / 2}^{t_{k}+T / 2} \exp (\mathcal{E}(t)) d t\right)$.

We extract $2 \mathrm{D}$ features from the sharpened images $\mathcal{L}\left(t_{k}\right)$ and $\mathcal{L}\left(t_{k+1}\right)$ instead of the original blurry images.

Forward and Backward Alignment. The feature tracking can drift over time. To reduce the tracking drifting, we apply the feature tracking method both forward from $\mathcal{L}\left(t_{k}\right)$ and backward from $\mathcal{L}\left(t_{k+1}\right)$. As illustrated in Fig. 3, the bidirectional tracking results are stitched by associating the closest backward feature position to each forward feature position at the central timestamp $\left(t_{k}+t_{k+1}\right) / 2$. The stitching is not applied if the 2D distance between the two associated locations is farther than a pre-defined threshold (four pixels). For the $h$-th stitched trajectory, we fit a B-spline curve to its discretely tracked 2D pixel locations in a batch and calculate a continuous event feature trajectory $\mathcal{T}(h)$.

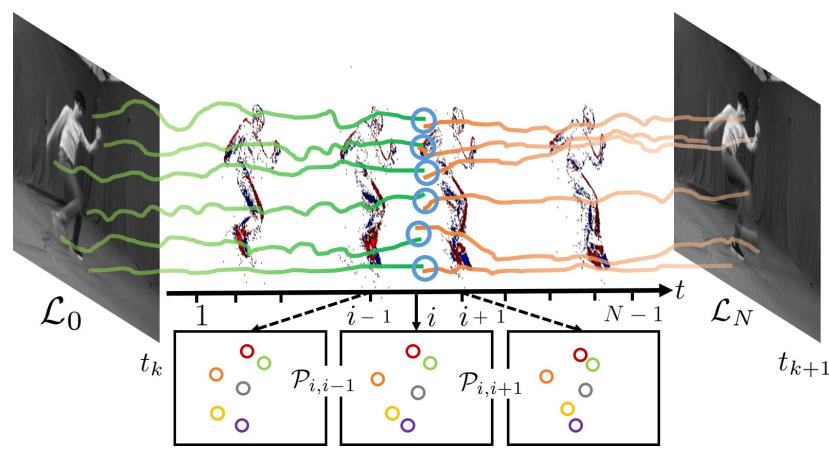

Figure 3: Illustration of asynchronous event trajectories between two adjacent intensity images. The green and orange curves represent the forward and backward event trajectories of exemplary photometric features. The blue circles denote alignment operation. The color-coded circles below indicate the 2D feature pairs between adjacent tracking frames.

Trajectory Slicing. In order to achieve motion capture at the desired tracking frame rate, e.g. $1000 \mathrm{fps}$, we evenly slice the continuous event trajectory $\mathcal{T}(h)$ at each millisecond time stamp (see Fig. 3). Since we perform tracking on each batch independently, for simplification we omit the subscript $k$ and let $0,1, \ldots, N$ denote the indexes of all the tracking frames for the current batch, where $N$ equals to the desired tracking frame rate divided by the frame rate of the intensity image stream. Thus, the intensity images $\mathcal{I}(k)$ and $\mathcal{I}(k+1)$ are denoted as $\mathcal{I}_{0}$ and $\mathcal{I}_{N}$ for short, and the corresponding latent images as $\mathcal{L}_{0}$ and $\mathcal{L}_{N}$.

\subsection{Hybrid Pose Batch Optimization}

Next, we jointly optimize all the skeleton poses $\mathcal{S}=$ $\left\{\mathbf{S}_{f}\right\}, f \in[0, N]$ for all the tracking frames in a batch. Our optimization leverages the hybrid input modality from the event camera. That is, we leverage not only the event feature correspondences obtained in Sec. 3.1, but also the CNN-based 2D and 3D pose estimates to tackle the drifting due to the accumulation of tracking errors and the inherent depth ambiguities of the monocular setting. We phrase the pose estimation across a batch as a constrained optimization problem:

$$
\begin{array}{ll}
\mathcal{S}^{*}=\underset{\mathcal{S}}{\arg \min } \boldsymbol{E}_{\text {batch }}(\mathcal{S}) \\
\text { s.t. } \quad \boldsymbol{\theta}_{\text {min }} \leq \boldsymbol{\theta}_{f} \leq \boldsymbol{\theta}_{\text {max }}, \quad \forall f \in[0, N],
\end{array}
$$

where $\boldsymbol{\theta}_{\min }$ and $\boldsymbol{\theta}_{\max }$ are the pre-defined lower and upper bounds of physically plausible joint angles to prevent unnatural poses. Our per-batch objective energy functional consists of four terms:

$$
\begin{aligned}
\boldsymbol{E}_{\text {batch }}(\mathcal{S})= & \lambda_{\text {adj }} \boldsymbol{E}_{\text {adj }}+\lambda_{2 \mathrm{D}} \boldsymbol{E}_{2 \mathrm{D}}+ \\
& \lambda_{3 \mathrm{D}} \boldsymbol{E}_{3 \mathrm{D}}+\lambda_{\text {temp }} \boldsymbol{E}_{\text {temp }} .
\end{aligned}
$$

Event Correspondence Term. The event correspondence term exploits the asynchronous spatio-temporal motion information encoded in the event stream. To this end, for the 
$i$-th tracking frame in a batch, we first extract the event correspondences from the sliced trajectories on two adjacent frames $i-1$ and $i+1$, as shown in Fig. 3. This forms two sets of event correspondences $\mathcal{P}_{i, i-1}$ and $\mathcal{P}_{i, i+1}$, where $\mathcal{P}_{i, *}=\left\{\left(p_{i, h}, p_{*, h}\right)\right\}, h \in[1, H]$. The term encourages the $2 \mathrm{D}$ projection of the template meshes to match the two sets of correspondences:

$\boldsymbol{E}_{\text {adj }}(\mathcal{S})=\sum_{i=1}^{N-1} \sum_{j \in\{i-1, i+1\}} \sum_{h=1}^{H} \tau\left(p_{i, h}\right)\left\|\pi\left(v_{i, h}\left(\mathbf{S}_{j}\right)\right)-p_{j, h}\right\|_{2}^{2}$,

where $\tau\left(p_{i, h}\right)$ is the indicator which equals to 1 only if the $2 \mathrm{D}$ pixel $p_{i, h}$ corresponds to a valid vertex of the mesh at the $i$-th tracking frame, and $v_{i, h}\left(\mathbf{S}_{j}\right)$ is the corresponding vertex on the mesh in pose $\mathbf{S}_{j}$.

2D and 3D Detection Terms. These terms encourage the posed skeleton to match the 2D and 3D body joint detection obtained by CNN from the intensity images. To this end, we apply VNect [35] and OpenPose [8] on the intensity images to estimate the $3 \mathrm{D}$ and $2 \mathrm{D}$ joint positions, denoted as $\mathbf{P}_{f, l}^{3 D}$ and $\mathbf{P}_{f, l}^{2 D}$, respectively, where $f \in\{0, N\}$ is the frame index, and $l$ is the joint index. Beside the body joints, We also use the four facial landmarks from the OpenPose [8] detection to recover the face orientation. The $2 \mathrm{D}$ term penalizes the differences between the projection of the landmarks of our model and the 2D detection:

$$
\boldsymbol{E}_{2 \mathrm{D}}(\mathcal{S})=\sum_{f \in\{0, N\}} \sum_{l=1}^{N_{J}+4}\left\|\pi\left(J_{l}\left(\mathbf{S}_{f}\right)\right)-\mathbf{P}_{f, l}^{2 D}\right\|_{2}^{2},
$$

where $J_{l}(\cdot)$ returns the 3D position of the $l$-th joint or face marker using the kinematic skeleton, and $\pi: \mathbb{R}^{3} \rightarrow \mathbb{R}^{2}$ is the perspective projection operator from $3 \mathrm{D}$ space to the $2 \mathrm{D}$ image plane. Our 3D term aligns the model joints and 3D detection:

$$
\boldsymbol{E}_{3 \mathrm{D}}(\mathcal{S})=\sum_{f \in\{0, N\}} \sum_{l=1}^{N_{J}}\left\|J_{l}\left(\mathbf{S}_{f}\right)-\left(\mathbf{P}_{f, l}^{3 D}+\mathbf{t}^{\prime}\right)\right\|_{2}^{2},
$$

where $\mathbf{t}^{\prime} \in \mathbb{R}^{3}$ is an auxiliary variable that transforms $\mathbf{P}_{f, l}^{3 D}$ from the root-centred to the global coordinate system [68].

Temporal Stabilization Term. Since only the moving body parts can trigger events, so far, the non-moving body parts are not constrained by our energy function. Therefore, we introduce a temporal stabilization constraint for the nonmoving body parts. This term penalizes the changes in joint positions between the current and previous tracking frames:

$$
\boldsymbol{E}_{\mathrm{temp}}(\mathcal{S})=\sum_{i=0}^{N-1} \sum_{l=1}^{N_{J}} \phi(l)\left\|J_{l}\left(\mathbf{S}_{i}\right)-J_{l}\left(\mathbf{S}_{i+1}\right)\right\|_{2}^{2}
$$

where the indicator $\phi(\cdot)$ equals to 1 if the corresponding body part is not associated with any event correspondence, and equals 0 otherwise.

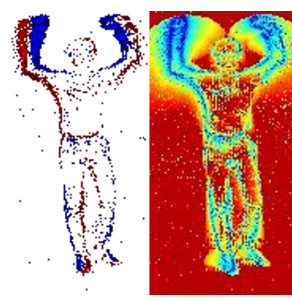

(a)

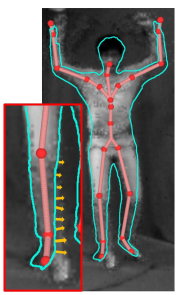

(b)

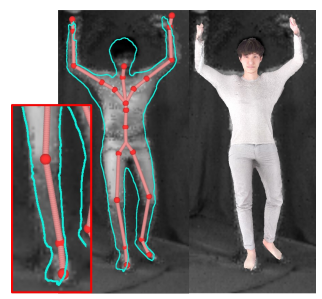

(c)
Figure 4: Event-based pose refinement. (a) Polarities and color-coded normalized distance map ranging from 0 (blue) to 1 (red). (b, c) The skeleton overlapped with the latent image before and after the refinement. Yellow arrows indicate the refined boundaries and exemplary 2D correspondences.

Optimization. We solve the constrained optimization problem (3) using the Levenberg-Marquardt (LM) algorithm of ceres [1]. For initialization, we minimize the 2D and 3D joint detection terms $\boldsymbol{E}_{2 \mathrm{D}}+\boldsymbol{E}_{3 \mathrm{D}}$ to obtain the initial values of $\mathbf{S}_{0}$ and $\mathbf{S}_{N}$, and then linearly interpolate $\mathbf{S}_{0}$ and $\mathbf{S}_{N}$ to obtain the initial values of all the tracking frames $\left\{\mathbf{S}_{f}\right\}$ in the current batch proportional to their timestamps.

\subsection{Event-Based Pose Refinement}

Most of the events are triggered by the moving edges in the image plane, which have a strong correlation with the actor's silhouette. Based on this finding, we refine our skeleton pose estimation in an Iterative Closest Point (ICP) [12] manner. In each ICP iteration, we first search for the closest event for each boundary pixel of the projected mesh. Then, we refine the pose $\mathbf{S}_{f}$ by solving the non-linear least squares optimization problem:

$$
\boldsymbol{E}_{\text {refine }}\left(\mathbf{S}_{f}\right)=\lambda_{\text {sil }} \boldsymbol{E}_{\text {sil }}\left(\mathbf{S}_{f}\right)+\lambda_{\text {stab }} \boldsymbol{E}_{\text {stab }}\left(\mathbf{S}_{f}\right)
$$

Here, we enforce the refined pose to stay close to its initial position using the following stability term:

$$
\boldsymbol{E}_{\mathrm{stab}}\left(\mathbf{S}_{f}\right)=\sum_{l=1}^{N_{J}}\left\|J_{l}\left(\mathbf{S}_{f}\right)-J_{i}\left(\hat{\mathbf{S}}_{f}\right)\right\|_{2}^{2}
$$

where $\hat{\mathbf{S}}_{f}$ is the skeleton pose after batch optimization (Sec. 3.2). The data term $\boldsymbol{E}_{\text {sil }}$ relies on the closest event search, which we will describe later. Let $s_{b}$ and $v_{b}$ denote the $b$ th boundary pixel and its corresponding 3D position on the mesh based on barycentric coordinates. For each $s_{b}$, let $u_{b}$ denote the corresponding target 2D position of the closest event. Then $\boldsymbol{E}_{\text {sil }}$ measures the 2D point-to-plane misalignment of the correspondences:

$$
\boldsymbol{E}_{\mathrm{sil}}\left(\mathbf{S}_{f}\right)=\sum_{b \in \mathcal{B}}\left\|\mathbf{n}_{b}^{\mathbf{T}}\left(\pi\left(v_{b}\left(\mathbf{S}_{f}\right)-u_{b}\right)\right)\right\|_{2}^{2},
$$

where $\mathcal{B}$ is the boundary set of the projected mesh and $\mathbf{n}_{b} \in$ $\mathbb{R}^{2}$ is the $2 \mathrm{D}$ normal vector corresponding to $s_{b}$. 

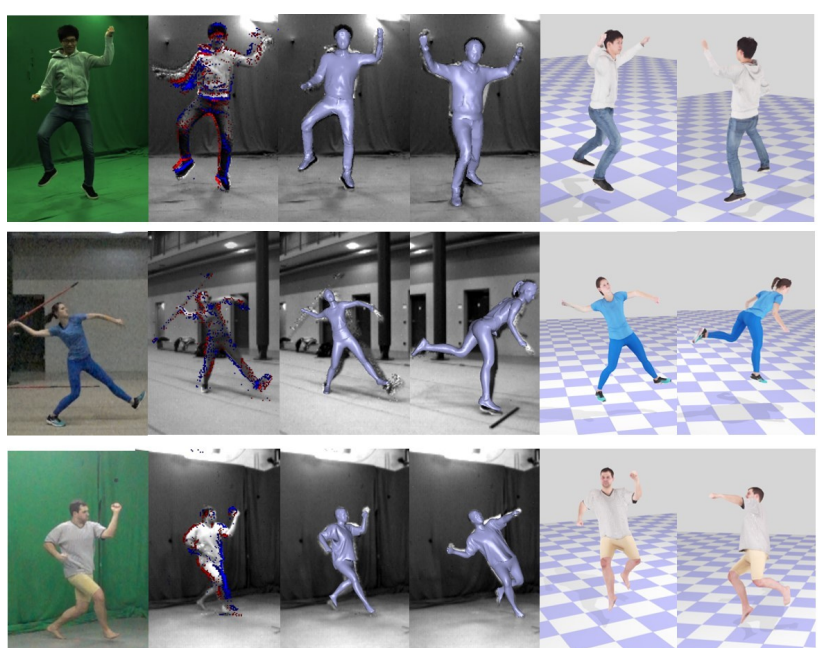

(a)

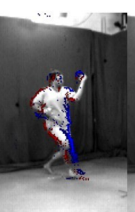

(b)

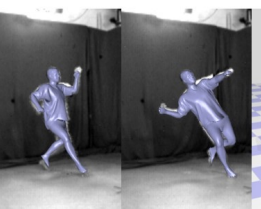

(c)

(d)

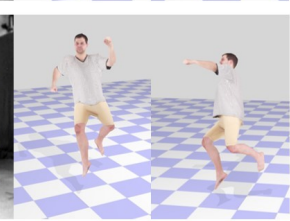

(e)

(f)
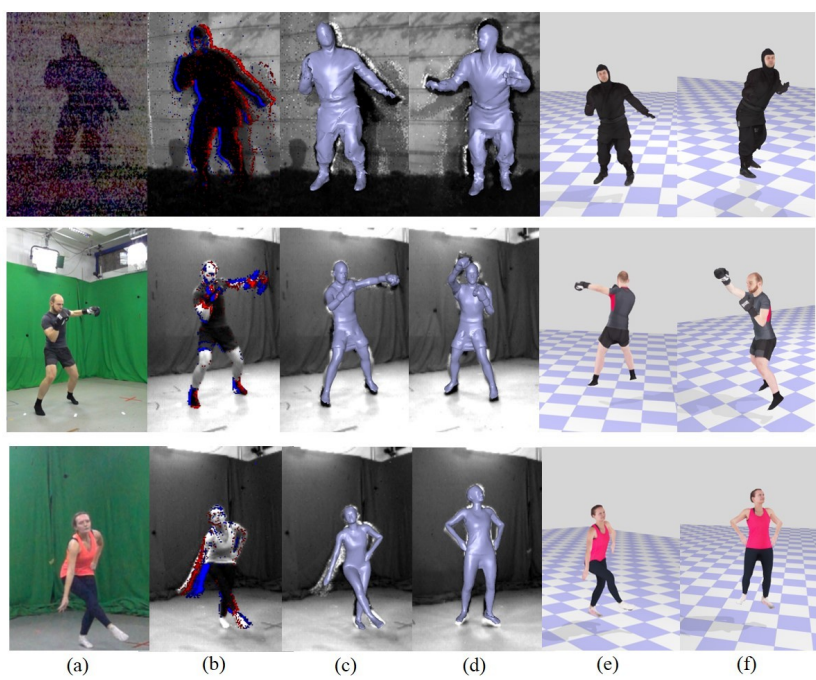

(a)

Figure 5: Qualitative results of EventCap on some sequences from our benchmark dataset, including "wave", "ninja", "javelin", "boxing", "karate" and "dancing" from the upper left to lower right. (a) The reference RGB image (not used for tracking); (b) Intensity images and the accumulated events; (c,d) Motion capture results overlaid on the reconstructed latent images; (e,f) Results rendered in 3D views.

Closest Event Search. Now we describe how to obtain the closest event for each boundary pixel $s_{b}$. The criterion for the closest event searching is based on the temporal and spatial distance between $s_{b}$ and each recent event $e=(u, t, \rho)$ :

$$
\mathcal{D}\left(s_{b}, e\right)=\lambda_{\text {dist }}\left\|\frac{t_{f}-t}{t_{N}-t_{0}}\right\|_{2}^{2}+\left\|s_{b}-u\right\|_{2}^{2},
$$

where $t_{f}$ is the timestamp of the current tracking frame, $\lambda_{\text {dist }}$ balances the weights of temporal and spatial distances, and $t_{N}-t_{0}$ equals to the time duration of a batch. We then solve the following local searching problem to obtain the closest event for each boundary pixel $s_{b}$ :

$$
e_{b}=\underset{e \in \mathcal{P}}{\arg \min } \mathcal{D}\left(s_{b}, e\right)
$$

Here, $\mathcal{P}$ is the collection of events, which happen within a local $8 \times 8$ spatial patch centred at $s_{b}$ and within the batchduration-sized temporal window centered at $t_{f}$. The position $u_{b}$ of the closest event $e_{b}$ is further utilized in Eq. (11).

Optimization. During the event-based refinement, we initialize $\mathbf{S}_{f}$ with the batch-based estimates and typically perform four ICP iterations. In each iteration, the energy in Eq. (9) is solved using the LM method provided by ceres [1]. As shown in Figs. 4(b) and 4(c), our iterative refinement based on the event stream improves the pose estimates.

\section{Experimental Results}

In this section, we evaluate our EventCap method on a variety of challenging scenarios. We run our experiments on a PC with $3.6 \mathrm{GHz}$ Intel Xeon E5-1620 CPU and 16GB RAM. Our unoptimized CPU code takes 4.5 minutes for a batch (i.e. 40 frames or $40 \mathrm{~ms}$ ), which divides to 30 seconds for the event trajectory generation, 1.5 minutes for the batch optimization and 2.5 minutes for the pose refinement. In all experiments, we use the following empirically determined parameters: $\lambda_{3 D}=1, \lambda_{2 D}=200, \lambda_{a d j}=50, \lambda_{t e m p}=80$, $\lambda_{\text {sil }}=1.0, \lambda_{\text {stab }}=5.0$, and $\lambda_{\text {dist }}=4.0$.

EventCap Dataset. To evaluate our method, we propose a new benchmark dataset for monocular event-based 3D motion capture, consisting of 12 sequences of 6 actors performing different activities, including karate, dancing, javelin throwing, boxing, and other fast non-linear motions. All our sequences are captured with a DAVIS240C event camera, which produces an event stream and a low frame rate intensity image stream (between 7 and $25 \mathrm{fps}$ ) at $240 \times 180$ resolution. For reference, we also capture the actions with a Sony RX0 camera, which produces a high frame rate (between 250 and $1000 \mathrm{fps}$ ) RGB videos at $1920 \times 1080$ resolution. In order to perform a quantitative evaluation, one sequence is also tracked with a multi-view markerless motion capture system [9] at $100 \mathrm{fps}$. We will make our dataset publicly available.

Fig. 5 shows several example frames of our EventCap results on the proposed dataset. For qualitative evaluation, we reconstruct the latent images at $1000 \mathrm{fps}$ from the event stream using the method of [40]. We can see in Fig. 5 that our results can be precisely overlaid on the latent images (cd), and that our reconstructed poses are plausible in 3D (ef). The complete motion capture results are provided in our supplementary video. From the $1000 \mathrm{fps}$ motion capture results, we can see that our method can accurately capture the high-frequency temporal motion details, which cannot be achieved by using standard low fps videos. Benefiting from the high dynamic range of the event camera, our method 


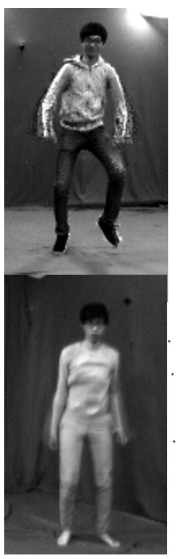

Intensity image

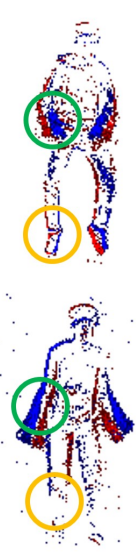

Events

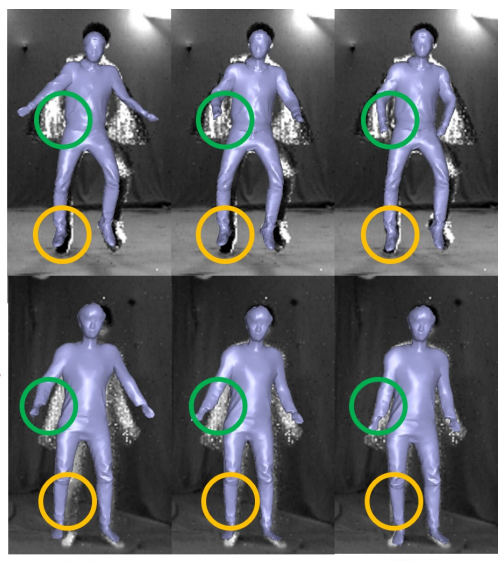

w/o batch

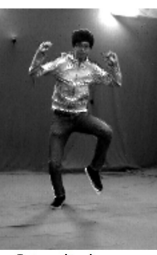

Intensity image

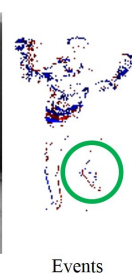

Events

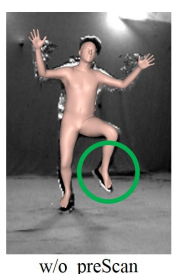

w/o_preScan

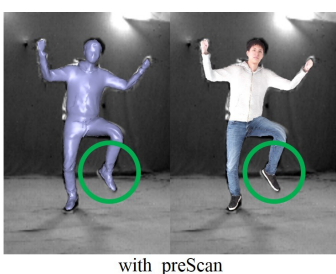

with_preScan
Figure 8: Influence of the template mesh accuracy. Our results using a prescanned template and using SMPL mesh are comparable, while the more accurate 3D scanned template improves the overlay on the latent images.

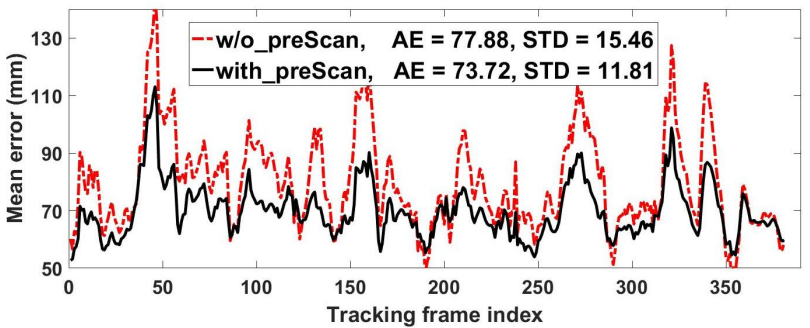

Figure 9: Quantitative analysis of the template mesh. The more accurate template improves the tracking accuracy in terms of average per-joint error.

maining misalignment, resulting in a better overlay on the reconstructed latent images. This is further evidenced by our quantitative evaluation in Fig. 7.

To this end, we obtain ground truth 3D joint positions using a multi-view markerless motion capture method [9]. Then, we compute the average per-joint error (AE) and the standard deviation (STD) of AE on every 10th tracking frame, because our tracking frame rate is $1000 \mathrm{fps}$ while the maximum capture frame rate of [9] is $100 \mathrm{fps}$. Following [68], to factor out the global pose, we perform Procrustes analysis to rigidly align our results to the ground truth. Fig. 7 shows our full pipeline consistently outperform the baselines on all frames, yielding both the lowest $\mathrm{AE}$ and the lowest STD. This not only highlights the contribution of each algorithmic component but also illustrates that our approach captures more high-frequency motion details in fast motions and achieves temporally more coherent results.

We further evaluate the influence of the template mesh accuracy. To this end, we compare the result using SMPL mesh from image-based body shape estimation [26] (denoted as w/o_preScan) against that using more accurate 3D scanned mesh (denoted as with_preScan). As shown in Fig. 8, the two methods yield comparable pose estimation results, while the 3D scanned mesh helps in terms of an image overlay since the SMPL mesh cannot model the clothes. Quantitatively, the method using 3D scanned mesh achieves a lower $\mathrm{AE}$ (73.72mm vs $77.88 \mathrm{~mm}$ ) as shown in Fig. 9.

\subsection{Comparison to Baselines}

To the best of our knowledge, our approach is the first monocular event-based 3D motion capture method. Therefore, we compare to existing monocular RGB-based approaches, HMR [26] and MonoPerfCap [68], which are 

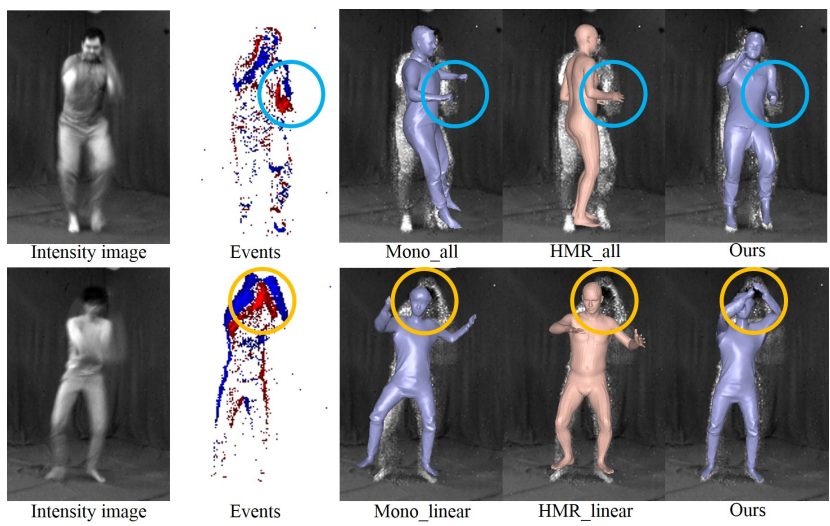

Figure 10: Qualitative comparison. Note that the polarity events are accumulated between the time duration from the previous to the current tracking frames. Our results overlay better with the latent images than the results of other methods.

most closely related to our approach. For a fair comparison, we first reconstruct the latent intensity images at $1000 \mathrm{fps}$ using [40]. Then, we apply HMR [27] and MonoPerfCap ${ }^{1}[68]$ on all latent images, denoted as HMR_all and Mono_all, respectively. We further apply MonoPerfCap [68] and HMR [27] only on the raw intensity images of low frame rate and linearly upsample the skeleton poses to $1000 \mathrm{fps}$, denoted as Mono_linear and HMR_linear, respectively. As shown in Fig. 10, both HMR_all and Mono_all suffer from inferior tracking results due to the accumulated error of the reconstructed latent images, while Mono_linear and HMR_linear fail to track the high-frequency motions. In contrast, our method achieves significantly better tracking results and more accurate overlay with the latent images. For quantitative comparison, we make use of the sequence with available ground truth poses (see Sec. 4.1). In Table 1, we report the mean $\mathrm{AE}$ of 1) all tracking frames (AE_all), 2) only the raw intensity frames (AE_raw), and 3) only the reconstructed latent image frames (AE_nonRaw). We also report the data throughput as the size of processed raw data per-second (Size_sec) for different methods. These quantitative results illustrate that our method achieves the highest tracking accuracy in our high frame rate setting. Furthermore, our method uses only $3.4 \%$ of the data bandwidth required in the high frame rate images setting (HMR_all and Mono_all), or only $10 \%$ higher compared to the low frame rate upsampling setting (Mono_linear and HMR_linear).

For further comparison, we apply MonoPerfCap [68] and HMR [27] to the high frame rate reference images directly, denoted as HMR_refer and Mono_refer, respectively. Due to the difference of image resolution between the reference and the event cameras, for a fair comparison, we downsample the reference images into the same resolution of the

\footnotetext{
${ }^{1}$ Only the pose optimization stage of MonoPerfCap is used, as their segmentation does not work well on the reconstructed latent images.
}

\begin{tabular}{|c|c|c|c||c|}
\hline & AE_all $(\mathrm{mm})$ & $\begin{array}{c}\text { AE_raw } \\
(\mathrm{mm})\end{array}$ & $\begin{array}{c}\text { AE_nonRaw } \\
(\mathrm{mm})\end{array}$ & $\begin{array}{c}\text { Size_sec } \\
(\mathrm{MB})\end{array}$ \\
\hline \hline Mono_linear & $88.6 \pm 17.3$ & $89.2 \pm 19.7$ & $88.5 \pm 16.8$ & $\mathbf{1 . 8 3}$ \\
\hline Mono_all & $98.4 \pm 22.8$ & $90.2 \pm 21.4$ & $99.8 \pm 23.0$ & 58.59 \\
\hline HMR_linear & $105.3 \pm 19.2$ & $104.3 \pm 20.6$ & $105.4 \pm 19.1$ & $\mathbf{1 . 8 3}$ \\
\hline HMR_all & $110.3 \pm 20.4$ & $105.5 \pm 19.5$ & $105.4 \pm 20.4$ & 58.59 \\
\hline Ours & $\mathbf{7 3 . 7} \pm \mathbf{1 1 . 8}$ & $\mathbf{7 5 . 2} \pm \mathbf{1 3 . 3}$ & $\mathbf{7 3 . 5} \pm \mathbf{1 1 . 3}$ & 2.02 \\
\hline
\end{tabular}

Table 1: Quantitative comparison of several methods in terms of tracking accuracy and data throughput.

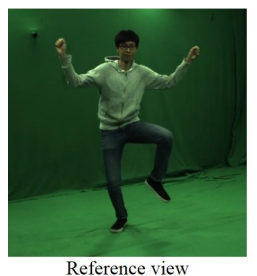

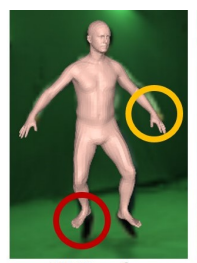

HMR refer

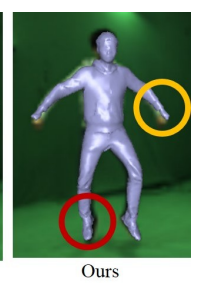

Ours
Figure 11: Qualitative comparison. Our results yield similar and even better overlay with the reference image, compared to results of Mono_refer and HMR_refer, respectively.

\begin{tabular}{|c|c|c|c|}
\hline & AE_all $(\mathrm{mm})$ & STD $(\mathrm{mm})$ & Size_sec $(\mathrm{MB})$ \\
\hline \hline Mono_refer & 76.5 & 13.4 & 58.59 \\
\hline HMR_refer & 83.5 & 17.8 & 58.59 \\
\hline Ours & $\mathbf{7 3 . 7}$ & $\mathbf{1 1 . 8}$ & $\mathbf{2 . 0 2}$ \\
\hline
\end{tabular}

Table 2: Quantitative comparison against Mono_refer and HMR_refer in terms of tracking accuracy and data throughput.

intensity image from the event camera. As shown in Fig. 11, our method achieves similar overlap to the reference image without using the high frame rate reference images. The corresponding AE and STD for all the tracking frames, as well as the Size _sec are reported in Table 2. Note that our method relies upon only $3.4 \%$ of the data bandwidth of the reference image-based methods, and even achieves better tracking accuracy compared to Mono_refer and HMR_refer.

\section{Discussion and Conclusion}

We present the first approach for markerless 3D human motion capture using a single event camera and a new dataset with high-speed human motions. Our batch optimization makes full usage of the hybrid image and event streams, while the captured motion is further refined with a new event-based pose refinement approach. Our experimental results demonstrate the effectiveness and robustness of EventCap in capturing fast human motions in various scenarios. We believe that it is a significant step to enable markerless capturing of high-speed human capture, with many potential applications in AR and VR, gaming, entertainment and performance evaluation for gymnastics, sports and dancing. In future work, we intend to investigate handling large occlusions and topological changes (e.g., opening a jacket) and improve the runtime performance. 


\section{References}

[1] S. Agarwal, K. Mierle, and Others. Ceres solver. http: // ceres-solver.org. 5, 6

[2] S. Amin, M. Andriluka, M. Rohrbach, and B. Schiele. Multiview pictorial structures for 3D human pose estimation. In British Machine Vision Conference (BMVC), 2009. 2

[3] A. Baak, M. Müller, G. Bharaj, H.-P. Seidel, and C. Theobalt. A data-driven approach for real-time full body pose reconstruction from a depth camera. In International Conference on Computer Vision (ICCV), 2011. 2

[4] F. Bogo, A. Kanazawa, C. Lassner, P. Gehler, J. Romero, and M. J. Black. Keep It SMPL: Automatic Estimation of 3D Human Pose and Shape from a Single Image. In European Conference on Computer Vision (ECCV), 2016. 2

[5] C. Bregler and J. Malik. Tracking people with twists and exponential maps. In Computer Vision and Pattern Recognition (CVPR), 1998. 2

[6] M. Burenius, J. Sullivan, and S. Carlsson. 3D pictorial structures for multiple view articulated pose estimation. In Computer Vision and Pattern Recognition (CVPR), 2013. 2

[7] E. Calabrese, G. Taverni, C. Awai Easthope, S. Skriabine, F. Corradi, L. Longinotti, K. Eng, and T. Delbruck. DHP19: Dynamic vision sensor 3d human pose dataset. In Computer Vision and Pattern Recognition (CVPR) Workshops, 2019. 3

[8] Z. Cao, T. Simon, S.-E. Wei, and Y. Sheikh. Realtime multiperson $2 \mathrm{~d}$ pose estimation using part affinity fields. In Computer Vision and Pattern Recognition (CVPR), 2017. 5

[9] The Captury. http://www. thecaptury.com/. 6, 7

[10] C.-H. Chen and D. Ramanan. $3 \mathrm{~d}$ human pose estimation $=2 \mathrm{~d}$ pose estimation + matching. In Computer Vision and Pattern Recognition (CVPR), 2016. 2

[11] W. Chen, H. Wang, Y. Li, H. Su, C. Tu, D. Lischinski, D. Cohen-Or, and B. Chen. Synthesizing training images for boosting human 3D pose estimation. In International Conference on $3 D$ Vision (3DV), 2016. 2

[12] Y. Chen and G. Medioni. Object modelling by registration of multiple range images. Image and Vision Computing (IVC), 10(3):145-155, 1992. 5

[13] A. J. Davison, J. Deutscher, and I. D. Reid. Markerless motion capture of complex full-body movement for character animation. In Eurographics Workshop on Computer Animation and Simulation, 2001. 1

[14] A. Elhayek, E. de Aguiar, A. Jain, J. Tompson, L. Pishchulin, M. Andriluka, C. Bregler, B. Schiele, and C. Theobalt. Efficient ConvNet-based marker-less motion capture in general scenes with a low number of cameras. In Computer Vision and Pattern Recognition (CVPR), 2015. 2

[15] L. Everding and J. Conradt. Low-latency line tracking using event-based dynamic vision sensors. Frontiers in Neurorobotics, 12:4, 2018. 3

[16] J. Gall, B. Rosenhahn, T. Brox, and H.-P. Seidel. Optimization and filtering for human motion capture. International Journal of Computer Vision (IJCV), 87(1-2):75-92, 2010. 2

[17] G. Gallego, T. Delbruck, G. Orchard, C. Bartolozzi, B. Taba, A. Censi, S. Leutenegger, A. Davison, J. Conradt, K. Daniilidis, and D. Scaramuzza. Event-based vision: A survey. arXiv e-prints, 2019. 2
[18] D. Gehrig, H. Rebecq, G. Gallego, and D. Scaramuzza. Asynchronous, photometric feature tracking using events and frames. In European Conference on Computer Vision (ECCV), 2018. 4

[19] K. Guo, J. Taylor, S. Fanello, A. Tagliasacchi, M. Dou, P. Davidson, A. Kowdle, and S. Izadi. Twinfusion: High framerate non-rigid fusion through fast correspondence tracking. In International Conference on $3 D$ Vision (3DV), pages 596-605, 2018. 2

[20] M. Habermann, W. Xu, M. Zollhöfer, G. Pons-Moll, and C. Theobalt. Livecap: Real-time human performance capture from monocular video. ACM Transactions on Graphics (TOG), 38(2):14:1-14:17, 2019. 2

[21] N. Hasler, B. Rosenhahn, T. Thormahlen, M. Wand, J. Gall, and H.-P. Seidel. Markerless motion capture with unsynchronized moving cameras. In Computer Vision and Pattern Recognition (CVPR), pages 224-231, 2009. 1

[22] M. B. Holte, C. Tran, M. M. Trivedi, and T. B. Moeslund. Human pose estimation and activity recognition from multiview videos: Comparative explorations of recent developments. Journal of Selected Topics in Signal Processing, 6(5):538-552, 2012. 2

[23] C. Ionescu, I. Papava, V. Olaru, and C. Sminchisescu. Human3.6M: Large Scale Datasets and Predictive Methods for 3D Human Sensing in Natural Environments. Transactions on Pattern Analysis and Machine Intelligence (TPAMI), 2014. 2

[24] E. Jahangiri and A. L. Yuille. Generating multiple hypotheses for human $3 \mathrm{~d}$ pose consistent with $2 \mathrm{~d}$ joint detections. In International Conference on Computer Vision (ICCV), 2017. 2

[25] H. Joo, H. Liu, L. Tan, L. Gui, B. Nabbe, I. Matthews, T. Kanade, S. Nobuhara, and Y. Sheikh. Panoptic studio: A massively multiview system for social motion capture. In International Conference on Computer Vision (ICCV), 2015. 2

[26] A. Kanazawa, M. J. Black, D. W. Jacobs, and J. Malik. Endto-end recovery of human shape and pose. In Computer $\mathrm{Vi}$ sion and Pattern Regognition (CVPR), 2018. 2, 3, 7, 8

[27] A. Kanazawa, M. J. Black, D. W. Jacobs, and J. Malik. Endto-end recovery of human shape and pose. In Computer Vision and Pattern Regognition (CVPR), 2018. 8

[28] N. Kolotouros, G. Pavlakos, and K. Daniilidis. Convolutional mesh regression for single-image human shape reconstruction. In Computer Vision and Pattern Recognition (CVPR), 2019. 2

[29] O. Kovalenko, V. Golyanik, J. Malik, A. Elhayek, and D. Stricker. Structure from Articulated Motion: An Accurate and Stable Monocular 3D Reconstruction Approach without Training Data. arXiv e-prints, 2019. 2

[30] A. Kowdle, C. Rhemann, S. Fanello, A. Tagliasacchi, J. Taylor, P. Davidson, M. Dou, K. Guo, C. Keskin, S. Khamis, D. Kim, D. Tang, V. Tankovich, J. Valentin, and S. Izadi. The need 4 speed in real-time dense visual tracking. In $S I G$ GRAPH Asia, pages 220:1-220:14, 2018. 1, 2

[31] S. Li and A. Chan. 3D Human Pose Estimation from Monocular Images with Deep Convolutional Neural Network. In Asian Conference on Computer Vision (ACCV), 2014. 2 
[32] P. Lichtsteiner, C. Posch, and T. Delbruck. A $128 \times 128120$ $\mathrm{db} 15 \mu$ s latency asynchronous temporal contrast vision sensor. IEEE Journal of Solid-State Circuits, 43(2):566-576, 2008. 1

[33] M. Loper, N. Mahmood, J. Romero, G. Pons-Moll, and M. J. Black. SMPL: A skinned multi-person linear model. In SIGGRAPH Asia, volume 34, pages 248:1-248:16, 2015. 3

[34] D. Mehta, H. Rhodin, D. Casas, P. Fua, O. Sotnychenko, $\mathrm{W}$. $\mathrm{Xu}$, and $\mathrm{C}$. Theobalt. Monocular 3d human pose estimation in the wild using improved cnn supervision. In International Conference on $3 D$ Vision (3DV), 2017. 2

[35] D. Mehta, S. Sridhar, O. Sotnychenko, H. Rhodin, M. Shafiei, H.-P. Seidel, W. Xu, D. Casas, and C. Theobalt. Vnect: Real-time 3d human pose estimation with a single rgb camera. ACM Transactions on Graphics (TOG), 36(4), 2017. 2, 5

[36] A. Mishra, R. Ghosh, A. Goyal, N. V. Thakor, and S. L. Kukreja. Real-time robot tracking and following with neuromorphic vision sensor. In International Conference on Biomedical Robotics and Biomechatronics (BioRob), 2016. 3

[37] T. B. Moeslund, A. Hilton, V. Krger, and L. Sigal, editors. Visual Analysis of Humans: Looking at People. Springer, 2011. 2

[38] Z. Ni, S.-H. Ieng, C. Posch, S. Rgnier, and R. Benosman. Visual tracking using neuromorphic asynchronous event-based cameras. Neural computation, 27:1-29, 02 2015. 3

[39] M. Omran, C. Lassner, G. Pons-Moll, P. V. Gehler, and B. Schiele. Neural body fitting: Unifying deep learning and model-based human pose and shape estimation. In International Conference on 3D Vision (3DV), 2018. 2

[40] L. Pan, C. Scheerlinck, X. Yu, R. Hartley, M. Liu, and Y. Dai. Bringing a blurry frame alive at high frame-rate with an event camera. arXiv e-prints, 2018. 4, 6, 8

[41] G. Pavlakos, X. Zhou, K. G. Derpanis, and K. Daniilidis. Coarse-to-fine volumetric prediction for single-image 3D human pose. In Computer Vision and Pattern Recognition (CVPR), 2017. 2

[42] G. Pavlakos, X. Zhou, K. G. Derpanis, and K. Daniilidis. Harvesting multiple views for marker-less $3 \mathrm{~d}$ human pose annotations. In Computer Vision and Pattern Recognition (CVPR), 2017. 2

[43] G. Pavlakos, L. Zhu, X. Zhou, and K. Daniilidis. Learning to estimate $3 \mathrm{D}$ human pose and shape from a single color image. In Computer Vision and Pattern Recognition (CVPR), 2018. 2

[44] Phasespace impulse x2e. http://phasespace.com/ x2e-motion-capture/. Accessed: 2019-07-05. 1, 2

[45] E. Piatkowska, A. N. Belbachir, S. Schraml, and M. Gelautz. Spatiotemporal multiple persons tracking using dynamic vision sensor. In Computer Vision and Pattern Recognition (CWPR) Workshops, pages 35-40, 2012. 3

[46] D. Reverter Valeiras, G. Orchard, S.-H. Ieng, and R. B. Benosman. Neuromorphic event-based 3d pose estimation. Frontiers in Neuroscience, 9:522, 2016. 3

[47] H. Rhodin, N. Robertini, C. Richardt, H.-P. Seidel, and C. Theobalt. A versatile scene model with differentiable vis- ibility applied to generative pose estimation. In International Conference on Computer Vision (ICCV), 2015. 2

[48] N. Robertini, D. Casas, H. Rhodin, H.-P. Seidel, and C. Theobalt. Model-based outdoor performance capture. In International Conference on 3D Vision (3DV), 2016. 2

[49] G. Rogez and C. Schmid. Mocap Guided Data Augmentation for 3D Pose Estimation in the Wild. In Neural Information Processing Systems (NIPS), 2016. 2

[50] J. Shotton, A. Fitzgibbon, M. Cook, T. Sharp, M. Finocchio, R. Moore, A. Kipman, and A. Blake. Real-time human pose recognition in parts from single depth images. In Computer Vision and Pattern Recognition (CVPR), 2011. 2

[51] L. Sigal, A. O. Bălan, and M. J. Black. HumanEva: Synchronized video and motion capture dataset and baseline algorithm for evaluation of articulated human motion. International Journal of Computer Vision (IJCV), 2010. 2

[52] L. Sigal, M. Isard, H. Haussecker, and M. J. Black. Looselimbed people: Estimating 3D human pose and motion using non-parametric belief propagation. International Journal of Computer Vision (IJCV), 98(1):15-48, 2012. 2

[53] T. Simon, H. Joo, I. Matthews, and Y. Sheikh. Hand keypoint detection in single images using multiview bootstrapping. In Computer Vision and Pattern Recognition (CVPR), 2017. 2

[54] C. Stoll, N. Hasler, J. Gall, H.-P. Seidel, and C. Theobalt. Fast articulated motion tracking using a sums of Gaussians body model. In International Conference on Computer Vision (ICCV), 2011. 1, 2

[55] V. Tan, I. Budvytis, and R. Cipolla. Indirect deep structured learning for $3 \mathrm{~d}$ human body shape and pose prediction. In British Machine Vision Conference (BMVC), 2018. 2

[56] B. Tekin, I. Katircioglu, M. Salzmann, V. Lepetit, and P. Fua. Structured Prediction of 3D Human Pose with Deep Neural Networks. In British Machine Vision Conference (BMVC), 2016. 2

[57] B. Tekin, P. Márquez-Neila, M. Salzmann, and P. Fua. Fusing 2D Uncertainty and 3D Cues for Monocular Body Pose Estimation. In International Conference on Computer Vision (ICCV), 2017. 2

[58] C. Theobalt, E. de Aguiar, C. Stoll, H.-P. Seidel, and S. Thrun. Performance capture from multi-view video. In Image and Geometry Processing for 3-D Cinematography, pages 127-149. Springer, 2010. 2

[59] Treedy's. https://www.treedys.com/. Accessed: 2019-07-25. 3

[60] D. R. Valeiras, X. Lagorce, X. Clady, C. Bartolozzi, S. Ieng, and R. Benosman. An asynchronous neuromorphic event-driven visual part-based shape tracking. Transactions on Neural Networks and Learning Systems (TNNLS), 26(12):3045-3059, 2015. 3

[61] G. Varol, J. Romero, X. Martin, N. Mahmood, M. Black, I. Laptev, and C. Schmid. Learning from synthetic humans. In Computer Vision and Pattern Recognition (CVPR), 2017. 2

[62] V. Vasco, A. Glover, E. Mueggler, D. Scaramuzza, L. Natale, and C. Bartolozzi. Independent motion detection with eventdriven cameras. In International Conference on Advanced Robotics (ICAR), pages 530-536, 2017. 3 
[63] Vicon Motion Systems. https://www.vicon.com/, 2019. 1, 2

[64] Y. Wang, Y. Liu, X. Tong, Q. Dai, and P. Tan. Outdoor markerless motion capture with sparse handheld video cameras. Transactions on Visualization and Computer Graphics (TVCG), 2017. 1

[65] X. Wei, P. Zhang, and J. Chai. Accurate realtime full-body motion capture using a single depth camera. SIGGRAPH Asia, 31(6):188:1-12, 2012. 2

[66] Xsens Technologies B.V. https://www.xsens.com/, 2019. 1,2

[67] L. Xu, Z. Su, L. Han, T. Yu, Y. Liu, and L. Fang. Unstructuredfusion: Realtime $4 \mathrm{~d}$ geometry and texture reconstruction using commercialrgbd cameras. Transactions on Pattern Analysis and Machine Intelligence (TPAMI), 2019. 2

[68] W. Xu, A. Chatterjee, M. Zollhöfer, H. Rhodin, D. Mehta, H.-P. Seidel, and C. Theobalt. Monoperfcap: Human performance capture from monocular video. ACM Transactions on Graphics (TOG), 37(2):27:1-27:15, 2018. 1, 2, 5, 7, 8

[69] H. Yasin, U. Iqbal, B. Kruger, A. Weber, and J. Gall. A Dual-Source Approach for 3D Pose Estimation from a Single Image. In Computer Vision and Pattern Recognition (CVPR), 2016. 2

[70] T. Yu, J. Zhao, Z. Zheng, K. Guo, Q. Dai, H. Li, G. PonsMoll, and Y. Liu. Doublefusion: Real-time capture of human performances with inner body shapes from a single depth sensor. Transactions on Pattern Analysis and Machine Intelligence (TPAMI), 2019. 2

[71] M.-Z. Yuan, L. Gao, H. Fu, and S. Xia. Temporal upsampling of depth maps using a hybrid camera. Transactions on Visualization and Computer Graphics (TVCG), 25(3):15911602, 2019. 1, 2

[72] X. Zhou, X. Sun, W. Zhang, S. Liang, and Y. Wei. Deep Kinematic Pose Regression. In European Conference on Computer Vision (ECCV) Workshops, 2016. 2

[73] X. Zhou, M. Zhu, S. Leonardos, K. Derpanis, and K. Daniilidis. Sparseness Meets Deepness: 3D Human Pose Estimation from Monocular Video. In Computer Vision and Pattern Recognition (CVPR), 2016. 2 